Check for updates

Cite this: J. Mater. Chem. A, 2019, 7, 11538

\section{Correction: Enabling room-temperature processed highly efficient and stable 2D Ruddlesden-Popper perovskite solar cells with eliminated hysteresis by synergistic exploitation of additives and solvents}

Shuang Yu, ${ }^{a}$ Yajie Yan, ${ }^{a}$ Yani Chen, ${ }^{a}$ Pavel Chábera, ${ }^{c}$ Kaibo Zheng ${ }^{\star b c}$ and Ziqi Liang*a

Correction for 'Enabling room-temperature processed highly efficient and stable 2D Ruddlesden-Popper perovskite solar cells with eliminated hysteresis by synergistic exploitation of additives and solvents' by Shuang Yu et al., J. Mater. Chem. A, 2019, 7, 2015-2021.

\title{
www.rsc.org/MaterialsA
}

The authors regret an error in the legend of Fig. $4 \mathrm{c}$ in the published article. A corrected version of Fig. 4 is shown below:

(a)

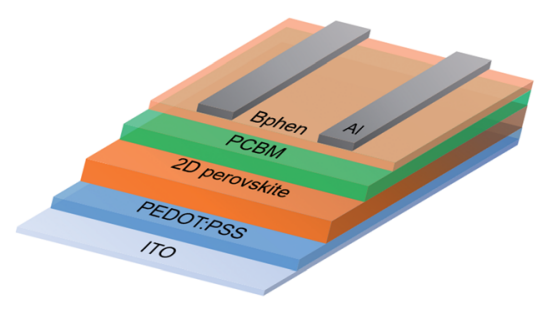

(c)

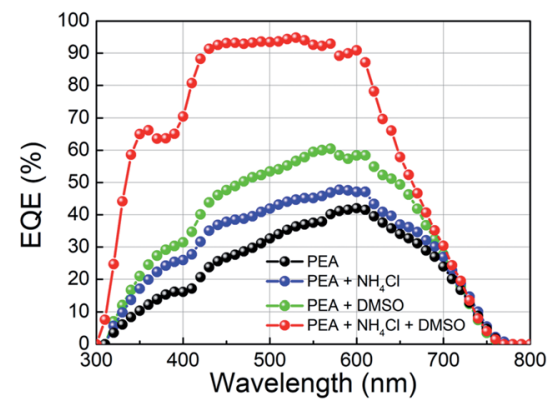

(b)
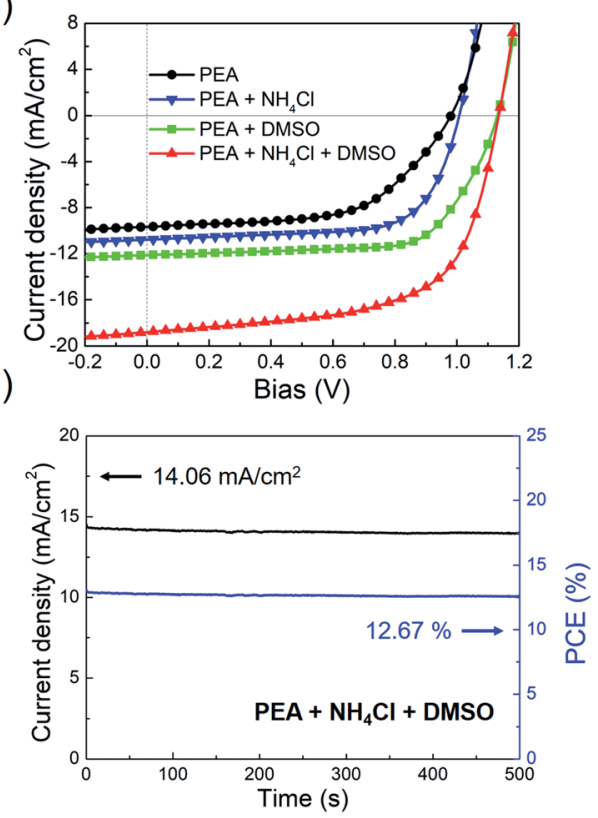

Fig. 4 (a) Schematic of solar cell device structures. (b) Representative current density-voltage ( $J-V$ ) characteristics of PEA perovskite based planar solar cells under a light irradiation of $100 \mathrm{~mW} \mathrm{~cm}^{-2}$ at reverse scan and their corresponding (c) EQE profiles. (d) Stabilized photocurrent density (black) and PCE (blue) of the optimal device based on PEA $+\mathrm{NH}_{4} \mathrm{Cl}+\mathrm{DMSO}$ over $500 \mathrm{~s}$ measured under a constant bias of $0.9 \mathrm{~V}$ near the maximum power point.

The Royal Society of Chemistry apologises for these errors and any consequent inconvenience to authors and readers.

${ }^{a}$ Department of Materials Science, Fudan University, Shanghai 200433, China. E-mail: zqliang@fudan.edu.cn

${ }^{b}$ Department of Chemistry, Technical University of Denmark, DK-2800 Kongens Lyngby, Denmark. E-mail: kzheng@kemi.dtu.dk

${ }^{c}$ Department of Chemical Physics and NanoLund, Lund University, Box 124, Lund, 22100, Sweden 